\title{
New Concepts for Surgical Management of Spontaneous Intra-Cerebral Hematomas
}

\author{
Abd El-Halim Abd-EIrazek Mousa, Mohamed Ahmed El-labbad, \\ Mustafa Mahmoud Mohamed Tolba*
}

Department of neurosurgery, Faculty of Medicine, Al-Azhar University, Cairo, Egypt

*Corresponding author: Mustafa Mahmoud Mohamed Tolba; Mobile: (+20)01019459503; Email: mustafatolba2@gmail.com

\begin{abstract}
Background: Spontaneous intracerebral hemorrhage $(\mathrm{ICH})$ represents cerebral parenchymal bleeding that may also extend into ventricular, and rarely, subarachnoid space. ICH, as a stroke subtype, is associated with poor neurological outcome as well as high mortality (about $40 \%$ per month). ICH can be classified as either primary or secondary, depending on the underlying cause of bleeding. Objective: This study was aimed to represent criteria for surgical management for spontaneous intracerebral hematomas. Patients and Methods: This study included a total of 30 subjects, both sexes, aged 19-80 years with variable sizes and sites of spontaneous intracerebral hematomas. Conscious level and functional disabilities were measured by the neurological examination, Radiological evaluation was done pre, early post-operative and a month later to evaluate: the evacuation of the hematoma, monitoring the hemostatic measures done intraoperative to prevent re-collection of the hematoma in presence of different co- morbidities like HTN, coagulopathy, ... etc. Results: Statistics revealed significant improvement in subjects operated with GCS $\geq 8$, with motor power deficit G 0 , clear chest , age below 60 years old $(\mathrm{P}>0.001)$.
\end{abstract}

Conclusion: Patients with spontaneous intracerebral hematomas showing good prognosis in patients below 60 years old, with clear chest complaining of motor power deficit G 0 , with GCS $\geq 8$.

Keywords: Surgical Management, Spontaneous Intra-Cerebral Hematomas.

\section{INTRODUCTION}

Spontaneous intracerebral hemorrhage (ICH) represents cerebral parenchymal bleeding that may also extend into ventricular, and rarely, subarachnoid space. $\mathrm{ICH}$, as a stroke subtype, is associated with poor neurological outcome as well as high mortality (about $40 \%$ per month $)^{(\mathbf{1})}$.ICH represents around $10-15 \%$ of all strokes in Europe, USA and Australia, while in Asia it accounts for about $20-30 \%$. It has been reported that every year 2 million people worldwide have $\mathrm{ICH}^{(\mathbf{1})}$. ICH can be classified as either primary or secondary, depending on the underlying cause of bleeding (2). Primary ICH is more common ( $78-88 \%$ of cases) and has its origin from chronic hypertension or amyloid angiopathy ${ }^{(3)}$.Secondary ICH is less common, and it is associated with vascular abnormalities (e.g. arteriovenous-malformations and aneurysms), impaired coagulation or tumors. Even though hypertensive ICH is the most frequent type of $\mathrm{ICH}$, vascular abnormalities should always be considered in these circumstances because of high risk of recurrent hemorrhage and also regarding the choice of the right therapy. Also, it should always be kept in mind that nowadays $\mathrm{ICH}$ as a consequence of the use of anticoagulant therapy is becoming increasingly frequent, and it occurs in almost $20 \%$ of cases in the USA ${ }^{(4)}$.

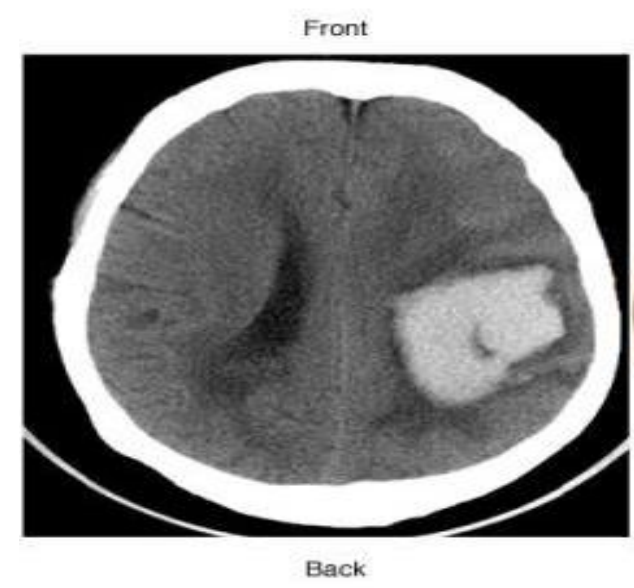

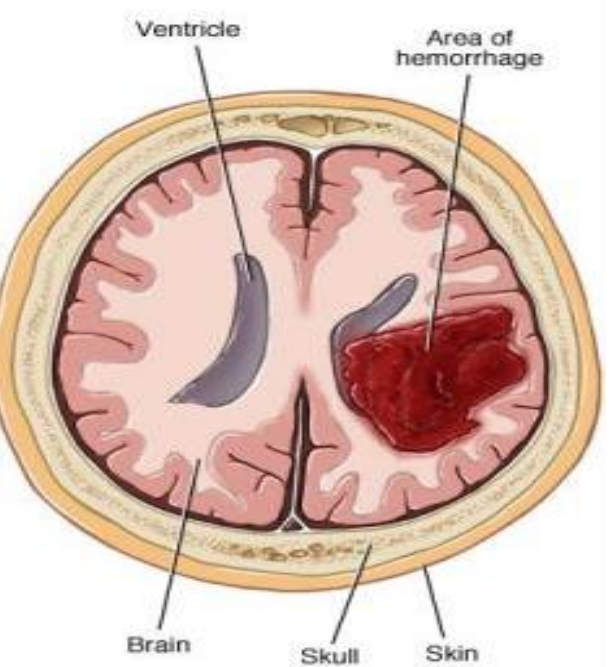

Fig. (1): CT brain showing lt intracerebral hematoma ${ }^{(4)}$. 
New Concepts for Surgical Management ...

\section{AIM OF THE WORK}

This study was aimed to represent criteria and prognosis for variable surgical managements for spontaneous intracerebral hematomas.

\section{PATIENTS AND METHODS}

This study included a total of 30 subjects, both sexes, aged 19-80 years with variable sizes and sites of spontaneous intracerebral hematomas, attending at AlAzhar University Hospitals.

Approval of the ethical committee and a written informed consent from all the subjects were obtained. This study was conducted between.

\section{Inclusion criteria}

- Age range 19 to 80 years.

- GCS $8 / 15$ or above.

- Clear chest.

- Motor power grade $0 / 5$.

- HTN.

- Liver impairment.

- Fits.

- Fit for surgery regarding to anesthesia

- Normal coagulation profile Exclusion criteria

- Age above 80 years.

- GCS less than 8/15.

- Bad chest condition.

- Motor power grade 2 or above.

- Site of HGE deep thalamic.

- Uncontrolled HTN.

- Uncorrectable bleeding profile.

Techniques:

Preoperative preparation:

- Good control of blood pressure to be $160 / 90$ or less

- Dehydrating measures to be received

- Good control of bleeding profile

- Antiepileptics to be received

Anesthesia

General anesthesia

- Open surgery:

First stage

Under hypotensive conditions:

Under good sterilization the craniotomy approach is determined according to the site of hematoma

Skin incision and bone flap achieved by perfoming burr holes

Elevation of the bone flap with the base towards the base of the skull

Dural insision
- Devascularization of the cortical incision prior to incision using the bipolar cautary

- Dissection of the cortical matter bluntly using the dissector.

- Making the track down to the site of hematoma (some times guided by brain needle first for confirmation).

- Evacuation of the hematoma side by side and placing patties over the evacuated areas

- After complete evacuation waiting for hemostasis for 10 minutes

$2^{\text {nd }}$ stage:

Removal of the placed patties, and placing absorbable hemostate (surgical of fibrillar)

And placing patties over them and waiting for 10 minutes for good hemostasis

$3^{\text {rd }}$ stage

Elevating the blood pressure to be normal or even slight elevated but not more than 160/90. And waiting for 10 minutes to confirm good hemostasis obtained

- Then closure in layer with under suction drain

Endoscopic surgery:

First stage:

Under hypotensive conditions

- Burr hole is determined to be at the center of the hematoma

- Linear dural incision is performed

- Devascularization of the site of the cortical incision by cautary of bibolar

- Introducing the endoscope trocher through the cortical incision using straight lens 30 and evacuation of the hematoma side by side and placing patties over the evacuated areas

- After complete evacuation waiting for hemostasis for 10 minutes

\section{$2^{\text {nd }}$ stage:}

Removal of the placed patties, and placing absorbable hemostate (surgical of fibrillar).

And placing patties over them and waiting for 10 minutes for good hemostasis.

$3^{\text {rd }}$ stage

Elevating the blood pressure to be normal or even slight elevated but not more than 160/90.

And waiting for 10 minutes to confirm good hemostasis obtained.

Then closure in layer.

\section{Postoperative follow up:}

The response for surgical intervention done regarding conscious level and motor power was recorded. Also the suspected complications were recorded in-details such as recollection of hematoma, deterioration of the conscious level. 


\section{RESULTS}

The study was performed on 30 patients to demonstrating the criteria for surgical option of the spontaneous intracerebral hematomas.
The performed techniques included open and endoscopic options. The cases underwent open surgery were $96.7 \%$, and cases underwent endoscopic surgery were $3.3 \%$.

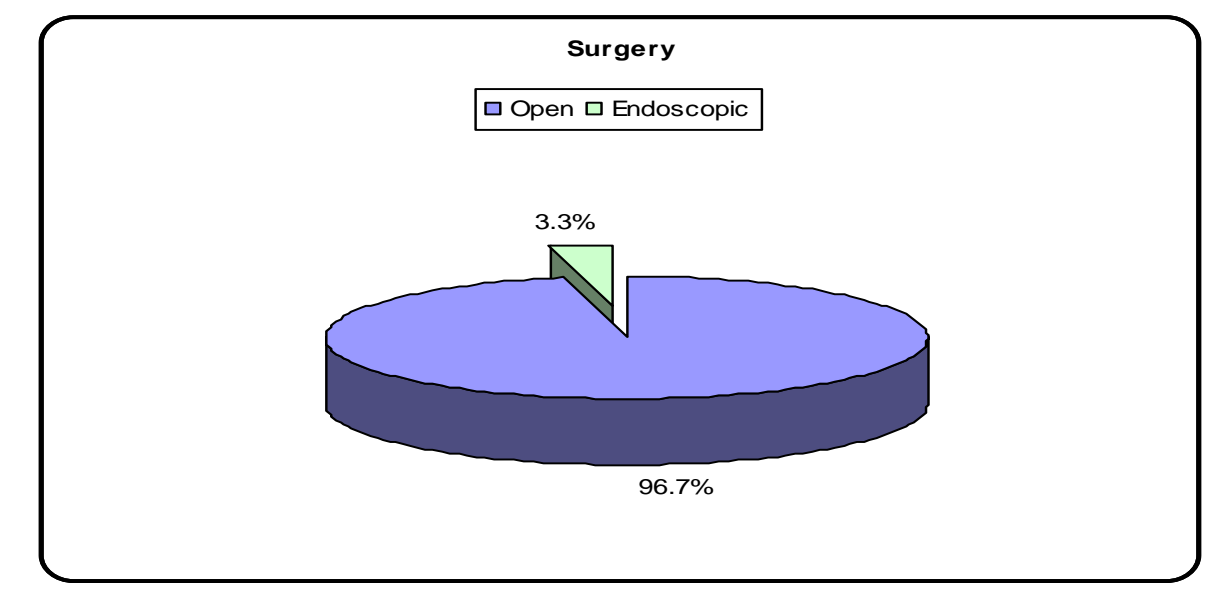

Fig. (2): The ratio of cases underwent open surgery 96.7 versus endoscopic surgery 3.3.

In the study, Patients with motor power grade 0 preoperative were $86.7 \%$ of cases Fig. (3).

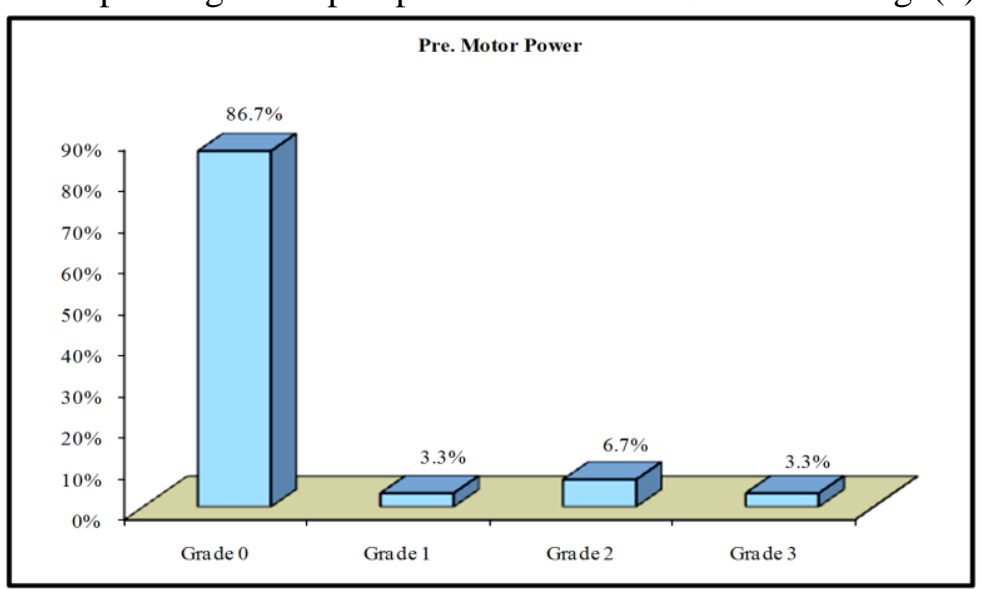

Fig. (3): Post-operative cases with motor power grade 0 reduced to be $40 \%$

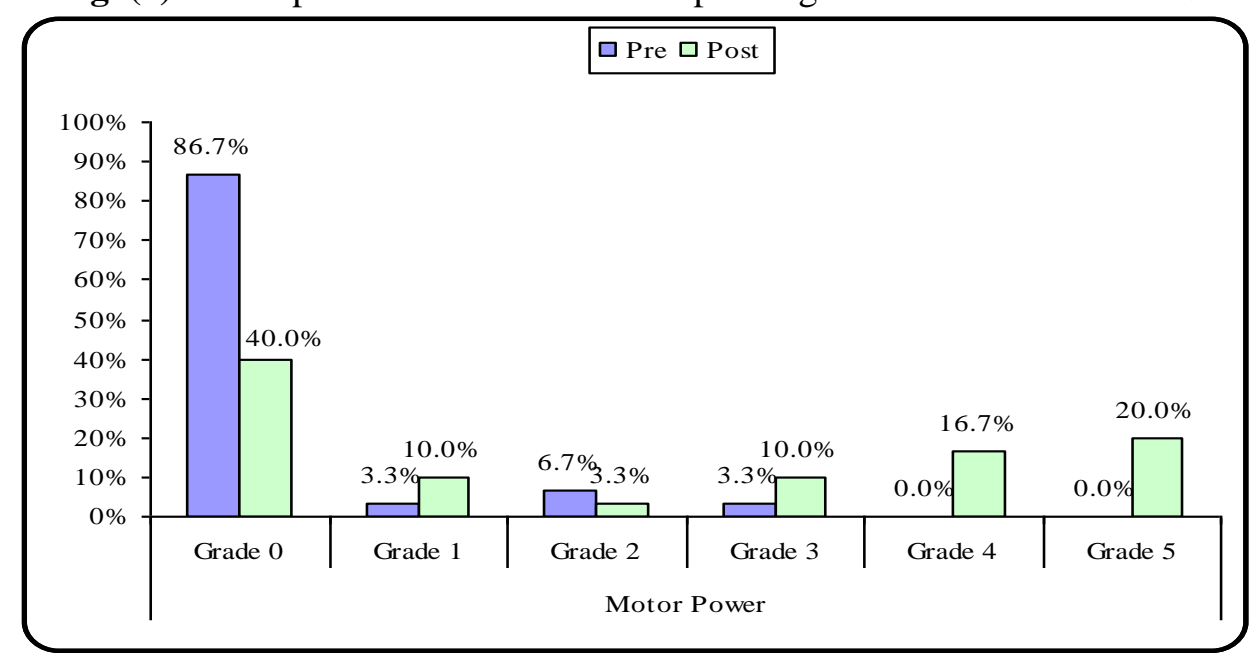

Fig. (4): Motor power grade pre and post treatment with reduction of cases with motor power G 0 from 86.7 to 40 . 
Table (1): The study showed improvement in GCS for patients underwent surgery with median GCS 8 / 15

\begin{tabular}{|c|l|c|c|c|c|c|}
\hline \multicolumn{2}{|c|}{} & Pre & Post & Test & P- & Sig. \\
\cline { 3 - 6 } & value & value & Sig. \\
\hline \multirow{2}{*}{ GCS } & $\begin{array}{l}\text { Median (IQR) } \\
\text { Range }\end{array}$ & $\begin{array}{c}8(7-11) \\
5-15\end{array}$ & $\begin{array}{c}12(3-15) \\
3-15\end{array}$ & $2.280 \bullet$ & 0.028 & S \\
\hline
\end{tabular}

The previous table shows that there was statistically significant increase in GCS post than pre with p-value. 0.028

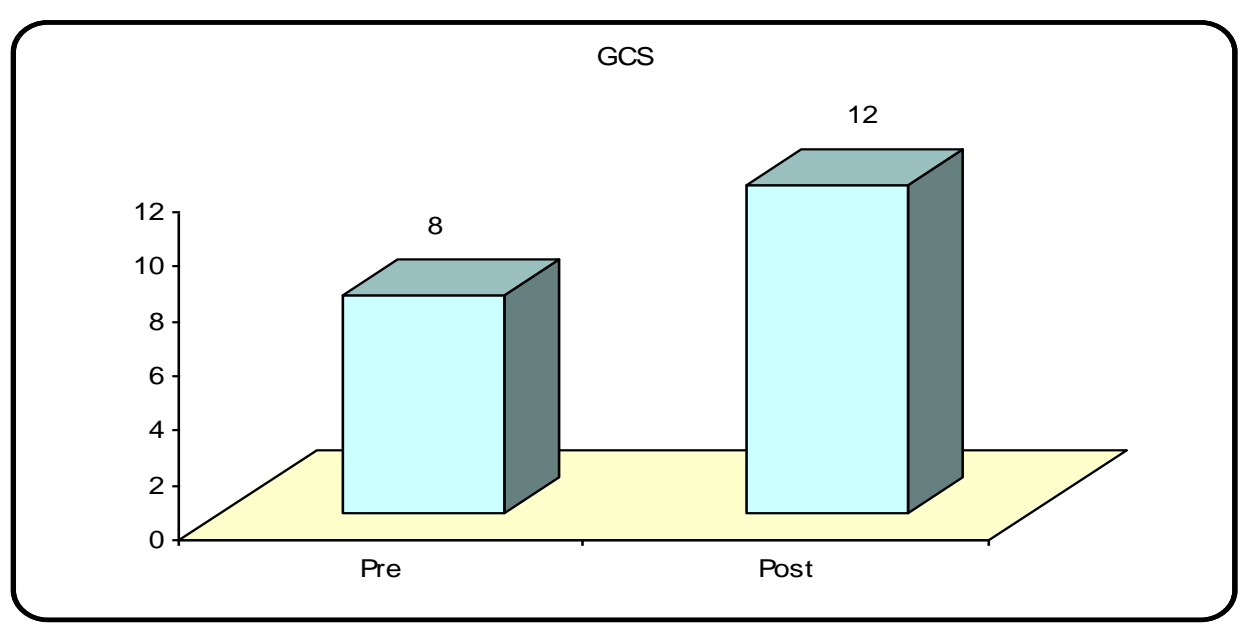

Fig. (5): GCS score pre and post treatment with improvement from 8 to 12 .

Study also showed that good results in surgical option could be achieved in patients less than 62 years.

\section{DISCUSSION}

The study is based on inclusion criteria that suppose good prognosis for patients with spontaneous intracerebral hematomas.

One of these criteria is the pre-operative GCS which is above $8 / 15$ in the study. The same result was in harmony with the American academy of neurology article published at $2006^{(5)}$.

\section{CONCLUSION}

Patients with spontaneous intracerebral hematomas showing good prognosis in patients below 60 years old, with clear chest complaining of motor power deficit G 0 , with GCS $\geq 8$.

\section{REFERENCES}

1. Keep RF, Hua Y and Xi G (2012): Intracerebral haemorrhage: mechanisms of injury and therapeutic targets. Lancet Neurol., 5:75-81.

2. Van Asch $\mathrm{C}$, Luitse $\mathrm{M}$, Rinkel $\mathrm{G}$ et al. (2010):Incidence, case fatality, and functional outcome of intracerebral haemorrhage over time, according to age, sex, and ethnic origin: a systematic review and meta-analysis. Lancet Neurol., 9: 167-76.

3. Qureshi AI, Tuhrim S, Broderick J et al. (2001): Spontaneous intracerebral hemorrhage. N Engl J Med., 344: $1450-60$.

4. Flaherty $\mathbf{M ( 2 0 1 0 ) :}$ Anticoagulant associated intracerebral hemorrhage.Semin Neurol ., 30: 565-72.

5. Davis SM, Broderick J, Hennerici M et al. (2006):Hematoma growth is a determinant of mortality and poor outcome after intracerebral hemorrhage. Neurology, 66(8):1175-1181. 